\title{
Variable order Mittag-Leffler fractional operators on isolated time scales and application to the calculus of variations*
}

\author{
Thabet Abdeljawad ${ }^{a}$ \\ tabdeljawad@psu.edu.sa \\ Raziye Mert ${ }^{b}$ \\ rmert@thk.edu.tr \\ Delfim F. M. Torres ${ }^{c}$ \\ delfim@ua.pt \\ ${ }^{a}$ Department of Mathematics and General Sciences, \\ Prince Sultan University, P. O. Box 66833, 11586 Riyadh, Saudi Arabia \\ ${ }^{b}$ Mechatronic Engineering Department, \\ University of Turkish Aeronautical Association, 06790 Ankara, Turkey \\ ${ }^{c}$ Center for Research and Development in Mathematics and Applications (CIDMA), \\ Department of Mathematics, University of Aveiro, 3810-193 Aveiro, Portugal
}

\begin{abstract}
We introduce new fractional operators of variable order on isolated time scales with Mittag-Leffler kernels. This allows a general formulation of a class of fractional variational problems involving variable-order difference operators. Main results give fractional integration by parts formulas and necessary optimality conditions of Euler-Lagrange type.
\end{abstract}

Keywords: fractional calculus on isolated time scales; variable order operators with Mittag-Leffler kernels; fractional sums and differences of variable order; summation by parts; variational principles on isolated time scales.

MSC 2010: 26A33; 26E70; 49K05.

\section{Introduction}

Fractional calculus is a generalization of ordinary differentiation and integration to an arbitrary non-integer order. It has been used effectively in the modeling of many problems in various fields of science and engineering, reflecting successfully the description of non-local properties of complex systems [9, 30. For the sake of finding more fractional operators with different kernels, recently several authors have introduced and studied new non-local derivatives with non-singular kernels and have applied them successfully to some real world problems [2, 3, 8, 16, 17, 23. What makes those fractional derivatives with Mittag-Leffler kernels more interesting is that their corresponding fractional integrals contain Riemann-Liouville fractional integrals as part of their structure. Moreover, such operators enable numerical analysts to develop more efficient algorithms in solving fractional dynamical systems by concentrating only on the coefficients of the differential equations rather than worrying about the singularity of the kernels, as in the case of classical fractional operators [7].

\footnotetext{
*This is a preprint of a paper whose final and definite form is with Springer, as a chapter book.
} 
In 1993, Samko and Ross investigated integrals and derivatives not of a constant but of variable order [28, 29, 31]. Afterwards, several pure mathematical and applicational papers contributed to the theory of variable order fractional calculus [6, 18, 19, 22, 26, 27]. Here we continue this line of research.

The article is organized as follows. In Section 2, we introduce new definitions of two different types of left and right nabla fractional sums of variable order, two different types of discrete versions of the left and right generalized fractional integral operators, together with two different types of fractional sums and differences of variable order in the sense of Atangana-Baleanu. Afterwards, in Section 3, we prove integration by parts formulas for Atangana-Baleanu fractional sums and differences with variable order. We end with Section 3 , applying our results to the calculus of variations.

\section{Fractional sums and differences of variable order}

The study of fractional calculus on time scales was initiated with the papers [10, 11, 12] and is now under strong development: see, e.g., [13, 14, 15, 25, 32. Here, inspired by the results of [3, 8], we introduce new nabla fractional operators of variable order on isolated time scales. The reader interested on the motivation and importance to consider variable order operators is referred to [34, 35, 36] and references therein.

Let $a, b \in \mathbb{R}$ with $b-a$ a positive integer. The sets $\mathbb{N}_{a},{ }_{b} \mathbb{N}$, and $\mathbb{N}_{a, b}$ are defined by

$$
\mathbb{N}_{a}=\{a, a+1, a+2, \ldots\}, \quad{ }_{b} \mathbb{N}=\{\ldots, b-2, b-1, b\}, \quad \mathbb{N}_{a, b}=\{a, a+1, a+2, \ldots, b\},
$$

respectively. Our operators use the concepts of rising function and discrete Mittag-Leffler function.

Definition 1 (Rising function [20]). (i) For a natural number $m$ and $t \in \mathbb{R}$, the $m$ rising (ascending) factorial of $t$ is defined by

$$
t^{\bar{m}}=\prod_{k=0}^{m-1}(t+k), \quad t^{\overline{0}}=1 .
$$

(ii) For any real number $\alpha$, the (generalized) rising function is defined by

$$
t^{\bar{\alpha}}=\frac{\Gamma(t+\alpha)}{\Gamma(t)}, \quad t \in \mathbb{R} \backslash\{\ldots,-2,-1,0\}, \quad 0^{\bar{\alpha}}=0 .
$$

Definition 2 (Nabla discrete Mittag-Leffler function [1, 4]). For $\lambda \in \mathbb{R},|\lambda|<1$ and $\alpha, \beta, z \in$ $\mathbb{C}$ with $\operatorname{Re}(\alpha)>0$, the nabla discrete Mittag-Leffler function is defined by

$$
E_{\overline{\alpha, \beta}}(\lambda, z)=\sum_{k=0}^{\infty} \lambda^{k} \frac{z^{\overline{k \alpha+\beta-1}}}{\Gamma(\alpha k+\beta)} .
$$

For $\beta=1$, we write

$$
E_{\bar{\alpha}}(\lambda, z) \triangleq E_{\overline{\alpha, 1}}(\lambda, z)=\sum_{k=0}^{\infty} \lambda^{k} \frac{z^{\overline{k \alpha}}}{\Gamma(\alpha k+1)} .
$$

To start, we define two different types of nabla fractional sums of variable order. 
Definition 3 (Left nabla fractional sums of order $\alpha(t)$ - types $I$ and $I I)$. Let $0<\alpha(t) \leq 1$ for all $t \in \mathbb{N}_{a}$. For a function $f: \mathbb{N}_{a} \rightarrow \mathbb{R}$,

1. the type I left nabla fractional sum of order $\alpha(t)$ is defined by

$$
{ }_{a} \nabla^{-\alpha(t)} f(t)=\frac{1}{\Gamma(\alpha(t))} \sum_{s=a+1}^{t}(t-\rho(s))^{\overline{\alpha(t)-1}} f(s), \quad t \in \mathbb{N}_{a+1}
$$

2. the type II left nabla fractional sum of order $\alpha(t)$ is defined by

$$
{ }_{a}^{*} \nabla^{-\alpha(t)} f(t)=\sum_{s=a+1}^{t}(t-\rho(s))^{\overline{\alpha(s)-1}} f(s) \frac{1}{\Gamma(\alpha(s))}, \quad t \in \mathbb{N}_{a+1} .
$$

Definition 4 (Right nabla fractional sums of order $\alpha(t)$ - types $I$ and $I I$ ). Let $0<\alpha(t) \leq 1$ for all $t \in{ }_{b} \mathbb{N}$. For a function $f:{ }_{b} \mathbb{N} \rightarrow \mathbb{R}$,

1. the type I right nabla fractional sum of order $\alpha(t)$ is defined by

$$
\nabla_{b}^{-\alpha(t)} f(t)=\frac{1}{\Gamma(\alpha(t))} \sum_{s=t}^{b-1}(s-\rho(t))^{\overline{\alpha(t)-1}} f(s), \quad t \in{ }_{b-1} \mathbb{N}
$$

2. the type II right nabla fractional sum of order $\alpha(t)$ is defined by

$$
{ }^{*} \nabla_{b}^{-\alpha(t)} f(t)=\sum_{s=t}^{b-1}(s-\rho(t))^{\overline{\alpha(s)-1}} f(s) \frac{1}{\Gamma(\alpha(s))}, \quad t \in{ }_{b-1} \mathbb{N} .
$$

Following [3], we now define two different discrete versions of the left and right generalized fractional integral operators.

Definition 5 (Discrete left generalized fractional integral operators - types $I$ and $I I$ ). Let $0<\alpha(t)<1 / 2$ for all $t \in \mathbb{N}_{a}$. For a function $\varphi: \mathbb{N}_{a} \rightarrow \mathbb{R}$,

1. the type I discrete left generalized fractional integral operator is defined by

$$
\boldsymbol{E}_{\overline{\alpha(t), 1}, \frac{-\alpha(t)}{1-\alpha(t)}, a^{+}} \varphi(t)=\frac{B(\alpha(t))}{1-\alpha(t)} \sum_{s=a+1}^{t} E_{\overline{\alpha(t)}}\left[\frac{-\alpha(t)}{1-\alpha(t)}, t-\rho(s)\right] \varphi(s), \quad t \in \mathbb{N}_{a+1}
$$

2. the type II discrete left generalized fractional integral operator is defined by

$$
\mathcal{E}_{\overline{\alpha(t), 1}, \frac{-\alpha(t)}{1-\alpha(t)}, a^{+}} \varphi(t)=\sum_{s=a+1}^{t} \frac{B(\alpha(s))}{1-\alpha(s)} E_{\overline{\alpha(s)}}\left[\frac{-\alpha(s)}{1-\alpha(s)}, t-\rho(s)\right] \varphi(s), \quad t \in \mathbb{N}_{a+1} .
$$

Definition 6 (Discrete right generalized fractional integral operators - types $I$ and $I I$ ). Let $0<\alpha(t)<1 / 2$ for all $t \in{ }_{b} \mathbb{N}$. For a function $\varphi:{ }_{b} \mathbb{N} \rightarrow \mathbb{R}$,

1. the type I discrete right generalized fractional integral operator is defined by

$$
\boldsymbol{E}_{\overline{\alpha(t), 1}, \frac{-\alpha(t)}{1-\alpha(t)}, b^{-}} \varphi(t)=\frac{B(\alpha(t))}{1-\alpha(t)} \sum_{s=t}^{b-1} E_{\overline{\alpha(t)}}\left[\frac{-\alpha(t)}{1-\alpha(t)}, s-\rho(t)\right] \varphi(s), \quad t \in_{b-1} \mathbb{N} ;
$$


2. the type II discrete right generalized fractional integral operator is defined by

$$
\mathcal{E}_{\overline{\alpha(t), 1}, \frac{-\alpha(t)}{1-\alpha(t)}, b^{-}} \varphi(t)=\sum_{s=t}^{b-1} \frac{B(\alpha(s))}{1-\alpha(s)} E_{\overline{\alpha(s)}}\left[\frac{-\alpha(s)}{1-\alpha(s)}, s-\rho(t)\right] \varphi(s), \quad t \in{ }_{b-1} \mathbb{N} .
$$

We now define two different types of fractional sums and differences of variable order in the sense of Atangana-Baleanu [8] (the so-called $A B$ operators).

Definition 7 (Left $A B$ nabla fractional sums of order $\alpha(t)$ - types $I$ and $I I$ ). Let $0<$ $\alpha(t) \leq 1$ for all $t \in \mathbb{N}_{a}$. For a function $f: \mathbb{N}_{a} \rightarrow \mathbb{R}$,

1. the type I left $A B$ nabla fractional sum of order $\alpha(t)$ is defined by

$$
\begin{aligned}
{ }_{a}^{A B} \nabla^{-\alpha(t)} f(t) & =\frac{1-\alpha(t)}{B(\alpha(t))} f(t)+\frac{\alpha(t)}{B(\alpha(t)) \Gamma(\alpha(t))} \sum_{s=a+1}^{t}(t-\rho(s))^{\overline{\alpha(t)-1}} f(s) \\
& =\frac{1-\alpha(t)}{B(\alpha(t))} f(t)+\frac{\alpha(t)}{B(\alpha(t))}{ }^{a} \nabla^{-\alpha(t)} f(t), \quad t \in \mathbb{N}_{a+1}
\end{aligned}
$$

2. the type II left AB nabla fractional sum of order $\alpha(t)$ is defined by

$$
\begin{aligned}
{ }_{a}^{* A B} \nabla^{-\alpha(t)} f(t) & =\frac{1-\alpha(t)}{B(\alpha(t))} f(t)+\sum_{s=a+1}^{t} \frac{\alpha(s)}{B(\alpha(s)) \Gamma(\alpha(s))}(t-\rho(s))^{\overline{\alpha(s)-1}} f(s) \\
& =\frac{1-\alpha(t)}{B(\alpha(t))} f(t)+{ }_{a}^{*} \nabla^{-\alpha(t)} \frac{\alpha f}{B \circ \alpha}(t), \quad t \in \mathbb{N}_{a+1} .
\end{aligned}
$$

Definition 8 (Right $A B$ nabla fractional sums of order $\alpha(t)$ - types $I$ and $I I$ ). Let $0<$ $\alpha(t) \leq 1$ for all $t \in{ }_{b} \mathbb{N}$. For a function $f:{ }_{b} \mathbb{N} \rightarrow \mathbb{R}$,

1. the type I right $A B$ nabla fractional sum of order $\alpha(t)$ is defined by

$$
\begin{aligned}
{ }^{A B} \nabla_{b}^{-\alpha(t)} f(t) & =\frac{1-\alpha(t)}{B(\alpha(t))} f(t)+\frac{\alpha(t)}{B(\alpha(t)) \Gamma(\alpha(t))} \sum_{s=t}^{b-1}(s-\rho(t))^{\overline{\alpha(t)-1}} f(s) \\
& =\frac{1-\alpha(t)}{B(\alpha(t))} f(t)+\frac{\alpha(t)}{B(\alpha(t))} \nabla_{b}^{-\alpha(t)} f(t), \quad t \in{ }_{b-1} \mathbb{N}
\end{aligned}
$$

2. the type II right $A B$ nabla fractional sum of order $\alpha(t)$ is defined by

$$
\begin{aligned}
{ }^{* A B} \nabla_{b}^{-\alpha(t)} f(t) & =\frac{1-\alpha(t)}{B(\alpha(t))} f(t)+\sum_{s=t}^{b-1} \frac{\alpha(s)}{B(\alpha(s)) \Gamma(\alpha(s))}(s-\rho(t))^{\overline{\alpha(s)-1}} f(s) \\
& =\frac{1-\alpha(t)}{B(\alpha(t))} f(t)+{ }^{*} \nabla_{b}^{-\alpha(t)} \frac{\alpha f}{B \circ \alpha}(t), \quad t \in{ }_{b-1} \mathbb{N} .
\end{aligned}
$$

Note that in Definitions 7 and 8 , if $\alpha(t) \equiv 0$, then we recover the initial function; if $\alpha(t) \equiv 1$, then we recover the ordinary sum.

Definition 9 (Left Riemann-Liouville $A B$ nabla fractional differences of order $\alpha(t)$ - types I and II). Let $0<\alpha(t)<1 / 2$ for all $t \in \mathbb{N}_{a}$. For a function $f: \mathbb{N}_{a} \rightarrow \mathbb{R}$, 
1. the type I left Riemann-Liouville AB nabla fractional difference of order $\alpha(t)$ is defined by

$$
{ }_{a}^{A B R} \nabla^{\alpha(t)} f(t)=\nabla \boldsymbol{E}_{\overline{\alpha(t), 1}, \frac{-\alpha(t)}{1-\alpha(t)}, a^{+}} f(t), \quad t \in \mathbb{N}_{a+1}
$$

2. the type II left Riemann-Liouville AB nabla fractional difference of order $\alpha(t)$ is defined by

$$
{ }_{a}^{A B R} \widehat{\nabla}^{\alpha(t)} f(t)=\nabla \mathcal{E} \overline{\overline{\alpha(t), 1}, \frac{-\alpha(t)}{1-\alpha(t)}, a^{+}} f(t), \quad t \in \mathbb{N}_{a+1} .
$$

Definition 10 (Right Riemann-Liouville $A B$ nabla fractional differences of order $\alpha(t)$ types I and II). Let $0<\alpha(t)<1 / 2$ for all $t \in{ }_{b} \mathbb{N}$. For a function $f:{ }_{b} \mathbb{N} \rightarrow \mathbb{R}$,

1. the type I right Riemann-Liouville AB nabla fractional difference of order $\alpha(t)$ is defined by

$$
{ }^{A B R} \nabla_{b}^{\alpha(t)} f(t)=-\Delta \boldsymbol{E}_{\overline{\alpha(t), 1}, \frac{-\alpha(t)}{1-\alpha(t)}, b^{-}} f(t), \quad t \in{ }_{b-1} \mathbb{N} ;
$$

2. the type II right Riemann-Liouville AB nabla fractional difference of order $\alpha(t)$ is defined by

$$
{ }^{A B R} \widehat{\nabla}_{b}^{\alpha(t)} f(t)=-\Delta \mathcal{E}_{\overline{\alpha(t), 1}, \frac{-\alpha(t)}{1-\alpha(t)}, b^{-}} f(t), \quad t \in{ }_{b-1} \mathbb{N} .
$$

Definition 11 (Left Caputo $A B$ nabla fractional differences of order $\alpha(t)$ - types I and II). Let $0<\alpha(t)<1 / 2$ for all $t \in \mathbb{N}_{a}$. For a function $f: \mathbb{N}_{a} \rightarrow \mathbb{R}$,

1. the type I left Caputo AB nabla fractional difference of order $\alpha(t)$ is defined by

$$
{ }_{a}^{A B C} \nabla^{\alpha(t)} f(t)=\boldsymbol{E}_{\overline{\alpha(t), 1}, \frac{-\alpha(t)}{1-\alpha(t)}, a^{+}} \nabla f(t), \quad t \in \mathbb{N}_{a+1} ;
$$

2. the type II left Caputo AB nabla fractional difference of order $\alpha(t)$ is defined by

$$
{ }_{a}^{A B C} \widehat{\nabla}^{\alpha(t)} f(t)=\mathcal{E}_{\overline{\alpha(t), 1}, \frac{-\alpha(t)}{1-\alpha(t)}, a^{+}} \nabla f(t), \quad t \in \mathbb{N}_{a+1} .
$$

Definition 12 (Right Caputo $A B$ nabla fractional differences of order $\alpha(t)$ - types I and II). Let $0<\alpha(t)<1 / 2$ for all $t \in{ }_{b} \mathbb{N}$. For a function $f:{ }_{b} \mathbb{N} \rightarrow \mathbb{R}$,

1. the type I right Caputo AB nabla fractional difference of order $\alpha(t)$ is defined by

$$
{ }^{A B C} \nabla_{b}^{\alpha(t)} f(t)=-\boldsymbol{E}_{\overline{\alpha(t), 1}, \frac{-\alpha(t)}{1-\alpha(t)}, b^{-}} \Delta f(t), \quad t \in{ }_{b-1} \mathbb{N} ;
$$

2. the type II right Caputo AB nabla fractional difference of order $\alpha(t)$ is defined by

$$
{ }^{A B C} \widehat{\nabla}_{b}^{\alpha(t)} f(t)=-\mathcal{E}_{\overline{\alpha(t), 1}, \frac{-\alpha(t)}{1-\alpha(t)}, b^{-}} \Delta f(t), \quad t \in{ }_{b-1} \mathbb{N} .
$$

Remark 1. If we replace $\alpha(t)$ in (11) and (3) by $\alpha(t-s)$ and replace each $\alpha(s)$ in (2) and (4) by $\alpha(t-s)$, then the $A B R$ and $A B C$ fractional differences with variable order can be expressed in convolution form. Similarly, if we replace $\alpha(t)$ in (5) and (7) by $\alpha(t-s)$ and replace each $\alpha(s)$ in (6) and (8) by $\alpha(t-s)$, then the second part of the $A B$ fractional integrals with variable order can be expressed in convolution form. 


\section{Summation by parts for variable order fractional operators}

Summation/integration by parts has a very important role in mathematics: see, e.g., [21, 24, 33. This is particularly true in the calculus of variations and optimal control, to prove necessary optimality conditions of Euler-Lagrange type (cf. proof of Theorem 3).

Lemma 1 (Integration by parts formula for nabla fractional sums of order $\alpha(t)$ ). Let $0<$ $\alpha(t) \leq 1$ for all $t \in \mathbb{N}_{a, b}$. For functions $f, g: \mathbb{N}_{a, b} \rightarrow \mathbb{R}$, we have

$$
\begin{aligned}
& \sum_{t=a+1}^{b-1} f(t){ }_{a} \nabla^{-\alpha(t)} g(t)=\sum_{t=a+1}^{b-1} g(t){ }^{*} \nabla_{b}^{-\alpha(t)} f(t) ; \\
& \sum_{t=a+1}^{b-1} f(t) \nabla_{b}^{-\alpha(t)} g(t)=\sum_{t=a+1}^{b-1} g(t){ }_{a}^{*} \nabla^{-\alpha(t)} f(t) .
\end{aligned}
$$

Proof. From Definition 3, and by changing the order of summation, we get

$$
\begin{aligned}
\sum_{t=a+1}^{b-1} f(t){ }_{a} \nabla^{-\alpha(t)} g(t) & =\sum_{t=a+1}^{b-1} f(t) \frac{1}{\Gamma(\alpha(t))} \sum_{s=a+1}^{t}(t-\rho(s))^{\overline{\alpha(t)-1}} g(s) \\
& =\sum_{s=a+1}^{b-1} g(s)\left(\sum_{t=s}^{b-1}(t-\rho(s))^{\overline{\alpha(t)-1}} f(t) \frac{1}{\Gamma(\alpha(t))}\right) \\
& =\sum_{s=a+1}^{b-1} g(s)^{*} \nabla_{b}^{-\alpha(t)} f(s) .
\end{aligned}
$$

The proof of the second assertion follows similarly.

Now, with the help of Lemma 1, we can prove the following integration by parts formula for $A B$ fractional sums of variable order.

Theorem 1 (Integration by parts formula for $A B$ nabla fractional sums of order $\alpha(t)$ ). Let $0<\alpha(t) \leq 1$ for all $t \in \mathbb{N}_{a, b}$. For functions $f, g: \mathbb{N}_{a, b} \rightarrow \mathbb{R}$, we have

$$
\begin{aligned}
& \sum_{t=a+1}^{b-1} f(t)_{a}^{A B} \nabla^{-\alpha(t)} g(t)=\sum_{t=a+1}^{b-1} g(t)^{* A B} \nabla_{b}^{-\alpha(t)} f(t) ; \\
& \sum_{t=a+1}^{b-1} f(t) \stackrel{* A B}{a} \nabla^{-\alpha(t)} g(t)=\sum_{t=a+1}^{b-1} g(t){ }^{A B} \nabla_{b}^{-\alpha(t)} f(t) .
\end{aligned}
$$


Proof. From Definition 7 and the first part of Lemma 1, we get

$$
\begin{aligned}
\sum_{t=a+1}^{b-1} f(t){ }_{a}^{A B} \nabla^{-\alpha(t)} g(t) & =\sum_{t=a+1}^{b-1} f(t) \frac{1-\alpha(t)}{B(\alpha(t))} g(t)+\sum_{t=a+1}^{b-1} f(t) \frac{\alpha(t)}{B(\alpha(t))}{ }^{a} \nabla^{-\alpha(t)} g(t) \\
& =\sum_{t=a+1}^{b-1} f(t) \frac{1-\alpha(t)}{B(\alpha(t))} g(t)+\sum_{t=a+1}^{b-1} g(t)^{*} \nabla_{b}^{-\alpha(t)} \frac{\alpha f}{B \circ \alpha}(t) \\
& =\sum_{t=a+1}^{b-1} g(t)\left(\frac{1-\alpha(t)}{B(\alpha(t))} f(t)+{ }^{*} \nabla_{b}^{-\alpha(t)} \frac{\alpha f}{B \circ \alpha}(t)\right) \\
& =\sum_{t=a+1}^{b-1} g(t)^{* A B} \nabla_{b}^{-\alpha(t)} f(t) .
\end{aligned}
$$

The proof of the second assertion is similar to the first one. It follows from Definition 7 and the second part of Lemma 1 .

Lemma 2. Let $0<\alpha(t)<1 / 2$ for all $t \in \mathbb{N}_{a, b}$. For functions $f, g: \mathbb{N}_{a, b} \rightarrow \mathbb{R}$, we have

$$
\begin{aligned}
& \sum_{t=a+1}^{b-1} f(t) \boldsymbol{E}_{\overline{\alpha(t), 1}, \frac{-\alpha(t)}{1-\alpha(t)}, a^{+}} g(t)=\sum_{t=a+1}^{b-1} g(t) \mathcal{E}_{\overline{\alpha(t), 1}, \frac{-\alpha(t)}{1-\alpha(t)}, b^{-}} f(t) ; \\
& \sum_{t=a+1}^{b-1} f(t) \mathcal{E}_{\overline{\alpha(t), 1}, \frac{-\alpha(t)}{1-\alpha(t)}, a^{+}} g(t)=\sum_{t=a+1}^{b-1} g(t) \boldsymbol{E}_{\overline{\alpha(t), 1}, \frac{-\alpha(t)}{1-\alpha(t)}, b^{-}} f(t) .
\end{aligned}
$$

Proof. From Definitions 5 and 6, and by changing the order of summation, we have

$$
\begin{aligned}
\sum_{t=a+1}^{b-1} f(t) \mathbf{E}_{\overline{\alpha(t), 1}, \frac{-\alpha(t)}{1-\alpha(t)}, a^{+}} g(t) & =\sum_{t=a+1}^{b-1} f(t) \frac{B(\alpha(t))}{1-\alpha(t)} \sum_{s=a+1}^{t} E_{\overline{\alpha(t)}}\left[\frac{-\alpha(t)}{1-\alpha(t)}, t-\rho(s)\right] g(s) \\
& =\sum_{s=a+1}^{b-1} g(s) \sum_{t=s}^{b-1} \frac{B(\alpha(t))}{1-\alpha(t)} E_{\overline{\alpha(t)}}\left[\frac{-\alpha(t)}{1-\alpha(t)}, t-\rho(s)\right] f(t) \\
& =\sum_{s=a+1}^{b-1} g(s) \mathcal{E}_{\overline{\alpha(s), 1}, \frac{-\alpha(s)}{1-\alpha(s)}, b^{-}} f(s) .
\end{aligned}
$$

The proof of the second assertion follows similarly.

Theorem 2. Let $0<\alpha(t)<1 / 2$ for all $t \in \mathbb{N}_{a, b}$. For functions $f, g: \mathbb{N}_{a, b} \rightarrow \mathbb{R}$, we have

$$
\begin{aligned}
& \sum_{t=a+1}^{b-1} f(t){ }_{a}^{A B C} \nabla^{\alpha(t)} g(t)=\left.g(t) \mathcal{E} \overline{\overline{\alpha(t), 1}, \frac{-\alpha(t)}{1-\alpha(t)}, b^{-}} f(t)\right|_{a} ^{b-1}+\sum_{t=a+1}^{b-1} g(t-1){ }^{A B R} \widehat{\nabla}_{b}^{\alpha(t)} f(t-1) ; \\
& \sum_{t=a+1}^{b-1} f(t){ }_{a}^{A B C} \widehat{\nabla}^{\alpha(t)} g(t)=\left.g(t) \boldsymbol{E}_{\overline{\alpha(t), 1}, \frac{-\alpha(t)}{1-\alpha(t)}, b^{-}} f(t)\right|_{a} ^{b-1}+\sum_{t=a+1}^{b-1} g(t-1){ }^{A B R} \nabla_{b}^{\alpha(t)} f(t-1) ; \\
& \sum_{t=a+1}^{b-1} f(t){ }^{A B C} \nabla_{b}^{\alpha(t)} g(t)=-\left.g(t) \mathcal{E}_{\overline{\alpha(t), 1}, \frac{-\alpha(t)}{1-\alpha(t)}, a^{+}} f(t)\right|_{a+1} ^{b}+\sum_{t=a+1}^{b-1} g(t+1){ }_{a}^{A B R} \widehat{\nabla}^{\alpha(t)} f(t+1) ;
\end{aligned}
$$




$$
\sum_{t=a+1}^{b-1} f(t){ }^{A B C} \widehat{\nabla}_{b}^{\alpha(t)} g(t)=-\left.g(t) \boldsymbol{E}_{\overline{\alpha(t), 1}, \frac{-\alpha(t)}{1-\alpha(t)}, a^{+}} f(t)\right|_{a+1} ^{b}+\sum_{t=a+1}^{b-1} g(t+1){ }_{a}^{A B R} \nabla^{\alpha(t)} f(t+1) .
$$

Proof. We will only prove the first assertion. The proof of the others follow similarly. From Definitions 10 and 11, the first part of Lemma 2 and the summation by parts formula from ordinary difference calculus, we get

$$
\begin{array}{rl}
\sum_{t=a+1}^{b-1} & f(t){ }_{a}^{A B C} \nabla^{\alpha(t)} g(t) \\
& =\sum_{t=a+1}^{b-1} f(t) \mathbf{E}_{\overline{\alpha(t), 1}, \frac{-\alpha(t)}{1-\alpha(t)}, a^{+}} \nabla g(t) \\
& =\sum_{t=a+1}^{b-1} \nabla g(t) \mathcal{E}_{\overline{\alpha(t), 1}, \frac{-\alpha(t)}{1-\alpha(t)}, b^{-}} f(t) \\
& =\left.g(t) \mathcal{E}_{\overline{\alpha(t), 1}, \frac{-\alpha(t)}{1-\alpha(t)}, b^{-}} f(t)\right|_{a} ^{b-1}-\sum_{t=a+1}^{b-1} g(t-1) \nabla \mathcal{E}_{\overline{\alpha(t), 1}, \frac{-\alpha(t)}{1-\alpha(t)}, b^{-}} f(t) \\
& =\left.g(t) \mathcal{E}_{\overline{\alpha(t), 1}, \frac{-\alpha(t)}{1-\alpha(t)}, b^{-}} f(t)\right|_{a} ^{b-1}-\sum_{t=a+1}^{b-1} g(t-1) \Delta \mathcal{E}_{\overline{\alpha(t), 1}, \frac{-\alpha(t)}{1-\alpha(t)}, b^{-}} f(t-1) \\
& =\left.g(t) \mathcal{E}_{\overline{\alpha(t), 1}, \frac{-\alpha(t)}{1-\alpha(t)}, b^{-}} f(t)\right|_{a} ^{b-1}+\sum_{t=a+1}^{b-1} g(t-1){ }^{A B R} \widehat{\nabla}_{b}^{\alpha(t)} f(t-1) .
\end{array}
$$

The proof is complete.

\section{Variable order fractional variational principles}

The fractional calculus of variations of variable-order is a subject under strong current development [5, 37]. However, to the best of our knowledge, available results are only for the continuous time scale $\mathbb{T}=\mathbb{R}$. Here we obtain the main result of a variational calculus, that is, an Euler-Lagrange necessary optimality condition, for the isolated time scale $\mathbb{T}=\mathbb{N}_{a+1, b-1}$.

Let $J$ be a functional of the form

$$
J(f)=\sum_{t=a+1}^{b-1} L\left(t, f^{\rho}(t),{ }_{a}^{A B C} \nabla^{\alpha(t)} f(t)\right),
$$

where $0<\alpha(t)<1 / 2$ for all $t \in \mathbb{N}_{a+1, b-1}, f: \mathbb{N}_{a, b-1} \rightarrow \mathbb{R}$ and $L: \mathbb{N}_{a+1, b-1} \times \mathbb{R} \times \mathbb{R} \rightarrow \mathbb{R}$.

Theorem 3. Let $f$ be a local extremum of J satisfying the boundary conditions

$$
f(a)=A, \quad f(b-1)=B .
$$

Then $f$ satisfies the Euler-Lagrange equation

$$
L_{1}^{\sigma}(t)+{ }^{A B R} \widehat{\nabla}_{b}^{\alpha(t)} L_{2}(t)=0, \quad t \in \mathbb{N}_{a+1, b-2},
$$

where $L_{1}=\frac{\partial L}{\partial f^{\rho}}$ and $L_{2}=\frac{\partial L}{\partial \underset{a}{A B C} \nabla^{\alpha(t)} f}$. 
Proof. Let $\varepsilon$ be a small real parameter and $\eta: \mathbb{N}_{a, b-1} \rightarrow \mathbb{R}$ be a function such that $\eta(a)=$ $\eta(b-1)=0$. Consider a variation of $f$, say $f+\varepsilon \eta$. Since the Caputo difference operator ${ }_{a}^{A B C} \nabla^{\alpha(t)}$ is linear, it follows that

$$
J(f+\varepsilon \eta)=\sum_{t=a+1}^{b-1} L\left(t, f^{\rho}(t)+\varepsilon \eta^{\rho}(t),{ }_{a}^{A B C} \nabla^{\alpha(t)} f(t)+\varepsilon_{a}^{A B C} \nabla^{\alpha(t)} \eta(t)\right) .
$$

Define $\hat{J}(\varepsilon)=J(f+\varepsilon \eta)$. Because $f$ is a local extremizer of $J, \hat{J}$ attains a local extremum at $\varepsilon=0$. Differentiating $\hat{J}(\varepsilon)$ at zero, we get

$\sum_{t=a+1}^{b-1} \eta^{\rho}(t) \frac{\partial L}{\partial f^{\rho}}\left(t, f^{\rho}(t),{ }_{a}^{A B C} \nabla^{\alpha(t)} f(t)\right)+{ }_{a}^{A B C} \nabla^{\alpha(t)} \eta(t) \frac{\partial L}{\partial_{a}^{A B C} \nabla^{\alpha(t)} f}\left(t, f^{\rho}(t),{ }_{a}^{A B C} \nabla^{\alpha(t)} f(t)\right)=0$.

Using the first integration by parts formula in Theorem 2, we have

$$
\begin{gathered}
\sum_{t=a+1}^{b-1} \eta^{\rho}(t)\left[\frac{\partial L}{\partial f^{\rho}}\left(t, f^{\rho}(t),{ }_{a}^{A B C} \nabla^{\alpha(t)} f(t)\right)+\left({ }^{A B R} \widehat{\nabla}_{b}^{\alpha(t)} \frac{\partial L}{\partial{ }_{a}^{A B C} \nabla^{\alpha(t)} f}\left(t, f^{\rho}(t),{ }_{a}^{A B C} \nabla^{\alpha(t)} f(t)\right)\right)(t-1)\right] \\
+\left.\eta(t)\left(\mathcal{E} \overline{\alpha(t), 1}, \frac{-\alpha(t)}{1-\alpha(t)}, b^{-} \frac{\partial L}{\partial{ }_{a}^{A B C} \nabla^{\alpha(t)} f}\left(t, f^{\rho}(t),{ }_{a}^{A B C} \nabla^{\alpha(t)} f(t)\right)\right)(t)\right|_{a} ^{b-1}=0 .
\end{gathered}
$$

Since $\eta(a)=\eta(b-1)=0$ and $\eta$ is arbitrary, it follows that

$$
\frac{\partial L}{\partial f^{\rho}}\left(t, f^{\rho}(t),{ }_{a}^{A B C} \nabla^{\alpha(t)} f(t)\right)+\left({ }^{A B R} \widehat{\nabla}_{b}^{\alpha(t)} \frac{\partial L}{\partial{ }_{a}^{A B C} \nabla^{\alpha(t)} f}\left(t, f^{\rho}(t),{ }_{a}^{A B C} \nabla^{\alpha(t)} f(t)\right)\right)(t-1)=0
$$

for all $t \in \mathbb{N}_{a+2, b-1}$.

Although we only consider here a class of fractional variable order variational problems (FVOVP), our Theorem 3 can be easily extended to many other related FVOVPs involving the new variable-order fractional differences introduced in Section 2. We trust that this observation will initiate some interest in further future developments.

\section{Acknowledgements}

Abdeljawad is grateful to Prince Sultan University for funding this work through research group Nonlinear Analysis Methods in Applied Mathematics (NAMAM), number RG-DES2017-01-17; Torres to the support of FCT within the R\&D unit CIDMA, UID/MAT/04106/2013.

\section{References}

[1] T. Abdeljawad, On delta and nabla Caputo fractional differences and dual identities, Discrete Dyn. Nat. Soc. 2013 (2013), Art. ID 406910, 12 pp. arXiv:1102.1625

[2] T. Abdeljawad and D. Baleanu, Discrete fractional differences with nonsingular discrete MittagLeffler kernels, Adv. Difference Equ. 2016 (2016), Paper No. 232, 18 pp.

[3] T. Abdeljawad and D. Baleanu, On fractional derivatives with exponential kernel and their discrete versions, Rep. Math. Phys. 80 (2017), no. 1, 11-27. arXiv:1606.07958 
[4] T. Abdeljawad, F. Jarad and D. Baleanu, A semigroup-like property for discrete Mittag-Leffler functions, Adv. Difference Equ. 2012, 2012:72, 7 pp.

[5] R. Almeida, D. Tavares and D. F. M. Torres, The variable-order fractional calculus of variations, SpringerBriefs in Applied Sciences and Technology, Springer, Cham, 2019. arXiv:1805.00720

[6] T. M. Atanackovic and S. Pilipovic, Hamilton's principle with variable order fractional derivatives, Fract. Calc. Appl. Anal. 14 (2011), no. 1, 94-109.

[7] A. Atangana, Fractional operators with constant and variable order with application to geohydrology, Academic Press, London, 2018.

[8] A. Atangana and D. Baleanu, New fractional derivatives with non-local and non-singular kernel: theory and application to heat transfer model, Therm. Sci. 20 (2016), no. 2, 763-769.

[9] D. Baleanu, K. Diethelm, E. Scalas and J. J. Trujillo, Fractional calculus, Series on Complexity, Nonlinearity and Chaos, 3, World Scientific Publishing Co. Pte. Ltd., Hackensack, NJ, 2012.

[10] N. R. O. Bastos, R. A. C. Ferreira and D. F. M. Torres, Necessary optimality conditions for fractional difference problems of the calculus of variations, Discrete Contin. Dyn. Syst. 29 (2011), no. 2, 417-437. arXiv:1007.0594

[11] N. R. O. Bastos, R. A. C. Ferreira and D. F. M. Torres, Discrete-time fractional variational problems, Signal Process. 91 (2011), no. 3, 513-524. arXiv:1005.0252

[12] N. R. O. Bastos, D. Mozyrska and D. F. M. Torres, Fractional derivatives and integrals on time scales via the inverse generalized Laplace transform, Int. J. Math. Comput. 11 (2011), J11, 1-9. arXiv:1012.1555

[13] B. Bayour and D. F. M. Torres, Complex-valued fractional derivatives on time scales, in Differential and difference equations with applications, 79-87, Springer Proc. Math. Stat., 164, Springer, 2016. arXiv:1511.02153

[14] N. Benkhettou, A. M. C. Brito da Cruz and D. F. M. Torres, Nonsymmetric and symmetric fractional calculi on arbitrary nonempty closed sets, Math. Methods Appl. Sci. 39 (2016), no. 2, 261-279. arXiv:1502.07277

[15] N. Benkhettou, A. Hammoudi and D. F. M. Torres, Existence and uniqueness of solution for a fractional Riemann-Liouville initial value problem on time scales, J. King Saud Univ. Sci. 28 (2016), no. 1, 87-92. arXiv:1508.00754

[16] M. Caputo and M. Fabrizio, A new definition of fractional derivative without singular kernel, Progr. Fract. Differ. Appl. 1 (2015), no. 2, 73-85.

[17] M. Caputo and M. Fabrizio, Applications of new time and spatial fractional derivatives with exponential kernels, Progr. Fract. Differ. Appl. 2 (2016), no. 1, 1-11.

[18] C. F. M. Coimbra, Mechanics with variable-order differential operators, Ann. Phys. 12 (2003), no. 11-12, 692-703.

[19] G. Diaz and C. F. M. Coimbra, Nonlinear dynamics and control of a variable order oscillator with application to the van der Pol equation, Nonlinear Dynam. 56 (2009), no. 1-2, 145-157.

[20] C. Goodrich and A. C. Peterson, Discrete fractional calculus, Springer, Cham, 2015.

[21] Y. Hang, Y. Liu, X. Xu, Y. Chen and S. Mo, Sensitivity analysis based on Markovian integration by parts formula, Math. Comput. Appl. 22 (2017), no. 4, Paper No. 40, 12 pp.

[22] C. F. Lorenzo and T. T. Hartley, Variable order and distributed order fractional operators, Nonlinear Dynam. 29 (2002), no. 1-4, 57-98.

[23] J. Losada and J. J. Nieto, Properties of a new fractional derivative without singular kernel, Progr. Fract. Differ. Appl. 1 (2015), no. 2, 87-92. 
[24] R. B. Nelsen, Proof without Words: Integration by Parts, Math. Mag. 64 (1991), no. 2, 130.

[25] B. Pei, Y. Xu, G. Yin and X. Zhang, Averaging principles for functional stochastic partial differential equations driven by a fractional Brownian motion modulated by two-time-scale Markovian switching processes, Nonlinear Anal. Hybrid Syst. 27 (2018), 107-124.

[26] L. E. S. Ramirez and C. F. M. Coimbra, On the selection and meaning of variable order operators for dynamic modeling, Int. J. Differ. Equ. 2010 (2010), Art. ID 846107, 16 pp.

[27] L. E. S. Ramirez and C. F. M. Coimbra, On the variable order dynamics of the nonlinear wake caused by a sedimenting particle, Phys. D 240 (2011), no. 13, 1111-1118.

[28] B. Ross and S. Samko, Fractional integration operator of variable order in the Hölder spaces $H^{\lambda(x)}$, Internat. J. Math. Math. Sci. 18 (1995), no. 4, 777-788.

[29] S. G. Samko, Fractional integration and differentiation of variable order, Anal. Math. 21 (1995), no. 3, 213-236.

[30] S. G. Samko, A. A. Kilbas and O. I. Marichev, Fractional integrals and derivatives, translated from the 1987 Russian original, Gordon and Breach Science Publishers, Yverdon, 1993.

[31] S. G. Samko and B. Ross, Integration and differentiation to a variable fractional order, Integral Transform. Spec. Funct. 1 (1993), no. 4, 277-300.

[32] K. Sheng, W. Zhang and Z. Bai, Positive solutions to fractional boundary-value problems with p-Laplacian on time scales, Bound. Value Probl. 2018, 2018:70.

[33] J. Tang, Computation of an infinite integral using integration by parts, Math. Methods Appl. Sci. 41 (2018), no. 3, 929-935.

[34] D. Tavares, R. Almeida and D. F. M. Torres, Caputo derivatives of fractional variable order: numerical approximations, Commun. Nonlinear Sci. Numer. Simul. 35 (2016), 69-87. arXiv:1511.02017

[35] D. Tavares, R. Almeida and D. F. M. Torres, Constrained fractional variational problems of variable order, IEEE/CAA J. Autom. Sin. 4 (2017), no. 1, 80-88. arXiv:1606.07512

[36] D. Tavares, R. Almeida and D. F. M. Torres, Fractional Herglotz variational problems of variable order, Discrete Contin. Dyn. Syst. Ser. S 11 (2018), no. 1, 143-154. arXiv:1703.09104

[37] D. Tavares, R. Almeida and D. F. M. Torres, Combined fractional variational problems of variable order and some computational aspects, J. Comput. Appl. Math. 339 (2018), 374-388. arXiv: 1704.06486 